\title{
A thorough analysis of the contribution of experimental, derived and sequence-based predicted protein-protein interactions for functional annotation of proteins S1 File
}

\footnotetext{
Stavros Makrodimitris ${ }^{1,2, \dagger}$, Marcel J.T. Reinders ${ }^{1,3}$, Roeland C.H.J. van Ham ${ }^{1,2}$

${ }^{1}$ Delft Bioinformatics Lab, Delft University of Technology, Delft, the Netherlands, ${ }^{2}$ Keygene N.V., Wageningen, the Netherlands and

${ }^{3}$ Leiden Computational Biology Center, Leiden University Medical Center, Leiden, the Netherlands.

${ }^{\dagger}$ To whom correspondence should be addressed.
} 


\section{Statistics of PPI networks}

The experimental PPI networks of the four species have very different degree distributions, as shown in Figure S1. Table S1 lists the number of total and unique protein-protein interactions (PPIs) that each data source includes for Saccharomyces cerevisae, Escherichia coli, Arabidoisis thaliana and Solanum lycopersicum.

(a) S. cerevisiae

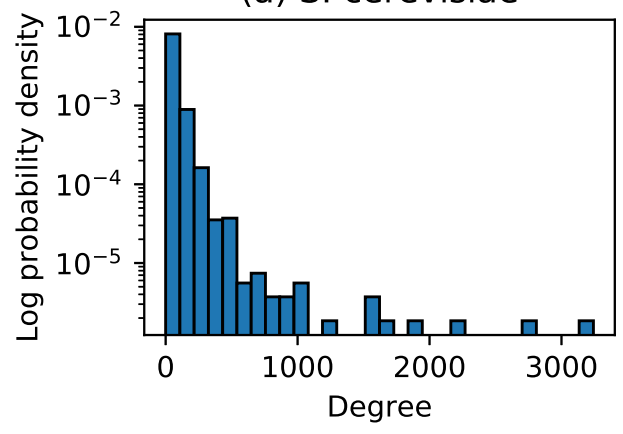

(c) A. thaliana

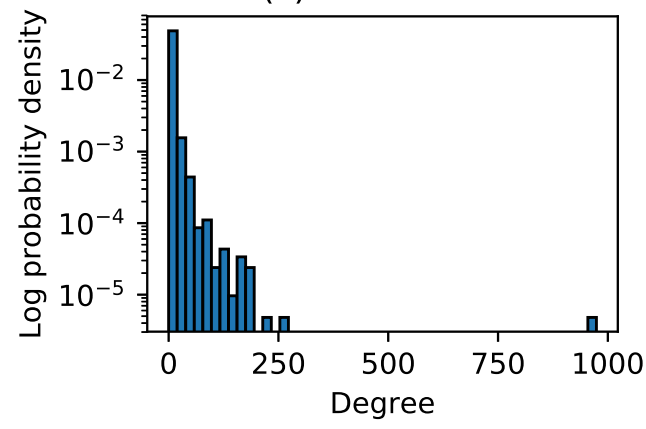

(b) E. coli

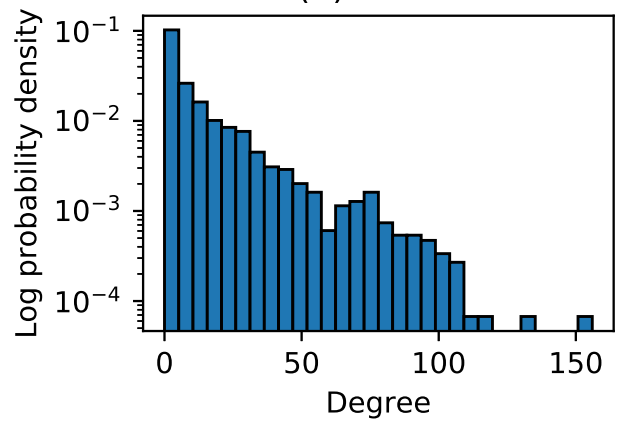

(d) S. Iycopersicum

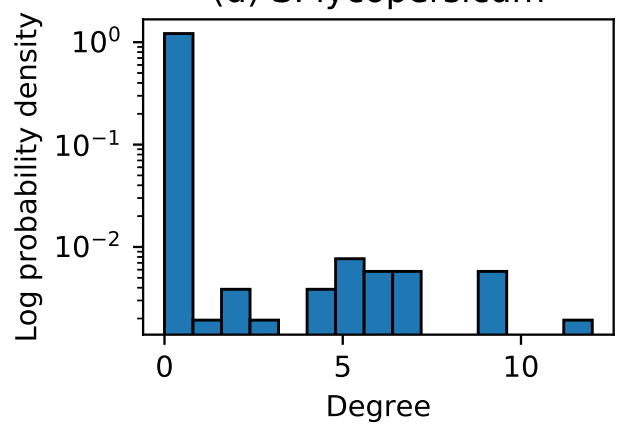

Figure S1: Histogram of node degrees in the experimental PPI networks of yeast (a), E. coli (b), arabidopsis (c) and tomato (d). On the $x$-axis node degree and on the $y$-axis the probability density in log scale. 
Table S1: Number of interactions added by each data source (rows) in each species (columns). "Database" from STRING was not considered, as it includes protein associations based on known GO annotations

\begin{tabular}{|l|c|c|c|c|c|c|c|c|}
\hline Data source & \multicolumn{2}{|c|}{ S. cerevisiae } & \multicolumn{2}{|c|}{ E. coli } & \multicolumn{2}{c|}{ A. thaliana } & \multicolumn{2}{c|}{ S. lycopersicum } \\
\hline & Total & Unique & Total & Unique & Total & Unique & Total & Unique \\
\hline $\begin{array}{l}\text { Physical (BI- } \\
\text { OGRID) }\end{array}$ & 97,503 & 51,473 & 9,734 & 5,295 & 21,720 & 10,826 & 57 & 11 \\
\hline $\begin{array}{l}\text { Experiments } \\
\text { (STRING) }\end{array}$ & 87,378 & 24,485 & 11,269 & 3,944 & 10,176 & 746 & 0 & 0 \\
\hline $\begin{array}{l}\text { Neighborhood } \\
\text { STRING) }\end{array}$ & 0 & 0 & 3,644 & 638 & 0 & 0 & 0 & 0 \\
\hline $\begin{array}{l}\text { Neighborhood } \\
\text { transferred } \\
\text { STRING) }\end{array}$ & 55,410 & 30,241 & 49,167 & 27,128 & 165,627 & 106,821 & 117 & 57 \\
\hline $\begin{array}{l}\text { Co-Occurence } \\
\text { (STRING) }\end{array}$ & 2,217 & 498 & 43,114 & 29,663 & 21,283 & 12,968 & 1,014 & 187 \\
\hline $\begin{array}{l}\text { Database trans- } \\
\text { ferred (STRING) }\end{array}$ & 0 & 0 & 0 & 0 & 0 & 0 & 0 & 0 \\
\hline $\begin{array}{l}\text { Experiments trans- } \\
\text { ferred (STRING) }\end{array}$ & 121,858 & 57,464 & 13,356 & 6,175 & 351,488 & 236,893 & 2,878 & 1,247 \\
\hline Fusion (STRING) & 1,784 & 1,007 & 1,568 & 224 & 2,010 & 671 & 2 & 0 \\
\hline $\begin{array}{l}\text { Homology } \\
\text { (STRING) }\end{array}$ & 3,700 & 561 & 1,083 & 414 & 31,983 & 16,734 & 1,858 & 212 \\
\hline $\begin{array}{l}\text { Co-Expression } \\
\text { (STRING) }\end{array}$ & 71,525 & 24,635 & 0 & 0 & 285,075 & 217,555 & 0 & 0 \\
\hline $\begin{array}{l}\text { Co-Expression } \\
\text { transferred } \\
\text { (STRING) }\end{array}$ & 242,228 & 100,849 & 47,897 & 21,878 & 390,727 & 193,802 & 3,029 & 622 \\
\hline $\begin{array}{l}\text { Text ming } \\
\text { (STRING) }\end{array}$ & 206,721 & 122,099 & 7,609 & 4,798 & 222,844 & 171,286 & 904 & 595 \\
\hline $\begin{array}{l}\text { Text mining trans- } \\
\text { ferred (STRING) }\end{array}$ & 211,409 & 95,945 & 56,745 & 30,854 & 497,495 & 313,994 & 3,712 & 1,412 \\
\hline
\end{tabular}




\section{Integration of STRING scores}

For completeness, we describe the algorithm for integrating STRING scores from $D$ different sources [1]. We use $s_{i}$ to denote the score (probability) of a particular interaction based on data type $i$. This score is computed by taking into account the prior probability of two proteins interacting $(p)$, for which the value $p=0.041$ is used. When combining multiple data types, this prior score should be only be incorporated once, so the first step is to remove it from all data types (Eq1):

$$
s_{i, \text { noprior }}=\frac{s_{i}-p}{1-p}
$$

Scores from different sources are combined using Eq2

$$
s_{\text {combined,noprior }}=1-\prod_{i=1}^{D}\left(1-s_{i, \text { noprior }}\right)
$$

Finally, the prior probability is incorporated back into the score by Eq 3 , which is the same as Eq[1, but solving for a different variable.

$$
s_{\text {combined }}=s_{\text {combined,noprior }}+p\left(1-s_{\text {combined,noprior }}\right)
$$




\section{Evaluation measures and results}

\section{Definitions}

We evaluated using the protein-centric F1 score and Semantic Distance $(S D)[2]$. For completeness, we provide definitions of these measures using notation similar to 3 : $T_{i}$ is the set of GO terms annotated for protein $i$ and $P_{i}(\tau)$ is the set of terms predicted for protein $i$ with posterior probability larger than $\tau$. $C$ denotes the number of GO terms and $n$ the number of proteins

F1 score is calculated from precision (equation 44 and recall (equation 5 from equation 6 . Semantic Distance is calculated based on Remaining Uncertainty ( $r u$, equation 7) and Misinformation ( $m i$, equation 8) using equation 9] [2]. ru quantifies the information content of the false negatives and misinformation that of the false positives.

$$
\begin{gathered}
\operatorname{pr}(\tau)=\frac{1}{n} \sum_{i=1}^{n} \frac{\sum_{t=1}^{C} I\left(t \in P_{i}(\tau) \wedge t \in T_{i}\right)}{\sum_{t=1}^{C} I\left(t \in P_{i}(\tau)\right)} \\
r c(\tau)=\frac{1}{n} \sum_{i=1}^{n} \frac{\sum_{t=1}^{C} I\left(t \in P_{i}(\tau) \wedge t \in T_{i}\right)}{\sum_{t=1}^{C} I\left(t \in T_{i}\right)} \\
F 1(\tau)=2 \frac{p r(\tau) \cdot r c(\tau)}{p r(\tau)+r c(\tau)} \\
r u(\tau)=\frac{1}{n} \sum_{i=1}^{n} I C(t) \cdot I\left(t \in T_{i} \wedge t \notin P_{i}(\tau)\right) \\
m i(\tau)=\frac{1}{n} \sum_{i=1}^{n} I C(t) \cdot I\left(t \notin T_{i} \wedge t \in P_{i}(\tau)\right) \\
S D(\tau)=\sqrt{r u(\tau)^{2}+m i(\tau)^{2}}
\end{gathered}
$$

All these measures are calculated using hard ( 0 or 1$)$ predictions and not posterior probabilities. Similar to the CAFA challenges [4], we report the metric value of each method for the optimal operating point $\tau$ (selected on the test data), considering all operating points from 0 to 1 with a step 0.02 (equations 10 and 11. Conversely to F1 score, lower Semantic Distance corresponds to better performance.

$$
\begin{aligned}
& F_{\text {max }}=\max _{\tau \in[0,1]} F 1(\tau) \\
& S_{\text {min }}=\min _{\tau \in[0,1]} S D(\tau)
\end{aligned}
$$

\section{Results}

The performance of all tested methods is listed in Tables $\mathrm{S2}$ and $\mathrm{S3}$

Table S2: $\mathrm{F}_{\max }$ of different function prediction methods (rows) in 4 species (columns) estimated using 5 -fold cross-validation. The mean \pm the standard deviation is shown.

\begin{tabular}{|l|l|l|l|l|}
\hline & S. cerevisiae & E. coli & A. thaliana & S. lycopersicum \\
\hline Naive & $0.31 \pm 0.004$ & $0.29 \pm 0.009$ & $0.28 \pm 0.005$ & $0.23 \pm 0.019$ \\
\hline BLAST & $0.35 \pm 0.004$ & $0.43 \pm 0.021$ & $0.42 \pm 0.007$ & $0.34 \pm 0.025$ \\
\hline EXP, GBA & $0.42 \pm 0.007$ & $0.25 \pm 0.011$ & $0.19 \pm 0.007$ & $0.08 \pm 0.007$ \\
\hline EXP, node2vec & $0.50 \pm 0.012$ & $0.28 \pm 0.011$ & $0.23 \pm 0.008$ & $0.08 \pm 0.042$ \\
\hline EXP, GBA + BLAST & $0.50 \pm 0.007$ & $0.45 \pm 0.019$ & $0.42 \pm 0.006$ & $0.33 \pm 0.025$ \\
\hline EXP + STRING, GBA & $0.49 \pm 0.005$ & $0.46 \pm 0.008$ & $0.48 \pm 0.004$ & $0.61 \pm 0.045$ \\
\hline EXP + STRING, node2vec & $0.59 \pm 0.009$ & $0.50 \pm 0.012$ & $0.50 \pm 0.005$ & $0.61 \pm 0.042$ \\
\hline EXP + STRING, GBA + BLAST & $0.54 \pm 0.006$ & $0.58 \pm 0.021$ & $0.54 \pm 0.008$ & $0.60 \pm 0.047$ \\
\hline EXP + SEQ, GBA & $0.33 \pm 0.005$ & $0.32 \pm 0.008$ & $0.29 \pm 0.004$ & $0.33 \pm 0.016$ \\
\hline
\end{tabular}


Table S3: $\mathrm{S}_{\min }$ of different function prediction methods (rows) in 4 species (columns) estimated using 5 -fold cross-validation. The mean \pm the standard deviation is shown.

\begin{tabular}{|l|l|l|l|l|}
\hline & S. cerevisiae & E. coli & A. thaliana & S. lycopersicum \\
\hline Naive & $34.67 \pm 0.75$ & $21.41 \pm 0.20$ & $30.44 \pm 0.57$ & $17.67 \pm 0.77$ \\
\hline BLAST & $35.24 \pm 0.97$ & $16.85 \pm 0.85$ & $27.66 \pm 0.31$ & $19.31 \pm 0.87$ \\
\hline EXP, GBA & $31.17 \pm 0.55$ & $21.58 \pm 0.44$ & $30.55 \pm 0.61$ & $19.39 \pm 0.91$ \\
\hline EXP, node2vec & $30.05 \pm 0.55$ & $20.37 \pm 0.41$ & $28.69 \pm 0.59$ & $19.37 \pm 0.89$ \\
\hline EXP, GBA + BLAST & $29.28 \pm 0.83$ & $15.57 \pm 0.79$ & $27.75 \pm 0.55$ & $18.25 \pm 0.92$ \\
\hline EXP + STRING, GBA & $27.42 \pm 0.57$ & $17.07 \pm 0.22$ & $24.12 \pm 0.50$ & $9.08 \pm 0.89$ \\
\hline EXP + STRING, node2vec & $25.65 \pm 0.48$ & $17.23 \pm 0.34$ & $25.10 \pm 0.15$ & $9.20 \pm 0.77$ \\
\hline EXP + STRING, GBA + BLAST & $28.48 \pm 0.97$ & $12.94 \pm 0.60$ & $23.00 \pm 0.56$ & $10.21 \pm 1.06$ \\
\hline EXP + SEQ, GBA & $34.50 \pm 0.74$ & $21.02 \pm 0.14$ & $30.48 \pm 0.57$ & $17.06 \pm 0.71$ \\
\hline
\end{tabular}

Table S4: $\mathrm{F}_{\max }, \mathrm{S}_{\min }$ and coverage of the node2vec method on the EXP PPI network in four species when excluding and including proteins without any functional annotations during the feature learning step. Where one of the two approaches is significantly (FDR $<5 \%$ ) better, it is shown in bold.

\begin{tabular}{|c|l|l|l|l|l|l|}
\hline Species & \multicolumn{2}{|c|}{$\mathrm{F}_{\max }$} & \multicolumn{2}{c|}{$\mathrm{S}_{\min }$} & \multicolumn{2}{c|}{ Coverage } \\
\hline & $\begin{array}{l}\text { Annotated } \\
\text { proteins only }\end{array}$ & All proteins & $\begin{array}{l}\text { Annotated } \\
\text { proteins only }\end{array}$ & All proteins & $\begin{array}{l}\text { Annotated } \\
\text { proteins only }\end{array}$ & All proteins \\
\hline S. cerevisiae & $0.50 \pm 0.012$ & $0.50 \pm 0.010$ & $30.05 \pm 0.54$ & $30.13 \pm 0.32$ & $0.99 \pm 0.002$ & $0.99 \pm 0.001$ \\
\hline E. coli & $0.28 \pm 0.011$ & $0.29 \pm 0.008$ & $20.37 \pm 0.41$ & $20.47 \pm 0.31$ & $0.77 \pm 0.016$ & $\mathbf{0 . 7 9} \pm \mathbf{0 . 0 1 7}$ \\
\hline A. thaliana & $0.23 \pm 0.008$ & $0.23 \pm 0.009$ & $28.69 \pm 0.49$ & $28.94 \pm 0.49$ & $0.57 \pm 0.010$ & $\mathbf{0 . 5 8} \pm \mathbf{0 . 0 1 1}$ \\
\hline S. lycopersicum & $0.08 \pm 0.007$ & $0.08 \pm 0.007$ & $19.37 \pm 0.89$ & $19.36 \pm 0.89$ & $0.03 \pm 0.017$ & $0.03 \pm 0.017$ \\
\hline
\end{tabular}

\section{Comparison of GBA and node2vec per protein}

Table S5: P-values before and after multiple testing correction to assess the significance in the median per-protein performances of GBA and node2vec at different levels of node degree. The bin numbers correspond to the bins shown in Figure 3 in the main document, with bin 1 denoting the smallest number of neighbors. For species where fewer bins were used, a dash signifies unused bins.

\begin{tabular}{|c|c|c|c|c|c|c|}
\hline Bin number & \multicolumn{2}{|c|}{ S. cerevisiae } & \multicolumn{2}{c|}{ E. coli } & \multicolumn{2}{c|}{ A. thaliana } \\
\hline & raw & corrected & raw & corrected & raw & corrected \\
\hline 1 & 0.028 & 0.036 & $7.5 \times 10^{-11}$ & $1.3 \times 10^{-10}$ & $<10^{-20}$ & $<10^{-20}$ \\
\hline 2 & $1.6 \times 10^{-5}$ & $2.9 \times 10^{-5}$ & $1.8 \times 10^{-11}$ & $9.0 \times 10^{-11}$ & $<10^{-20}$ & $<10^{-20}$ \\
\hline 3 & 0.845 & 0.845 & $7.6 \times 10^{-11}$ & $1.3 \times 10^{-10}$ & $<10^{-20}$ & $<10^{-20}$ \\
\hline 4 & 0.004 & 0.006 & 0.031 & 0.031 & $4.4 \times 10^{-16}$ & $5.5 \times 10^{-16}$ \\
\hline 5 & $7.4 \times 10^{-6}$ & $1.7 \times 10^{-5}$ & $1.9 \times 10^{-5}$ & $2.4 \times 10^{-5}$ & 0.001 & 0.001 \\
\hline 6 & $2.5 \times 10^{-21}$ & $2.2 \times 10^{-20}$ & - & - & - & - \\
\hline 7 & $5.5 \times 10^{-12}$ & $2.5 \times 10^{-11}$ & - & - & - & - \\
\hline 8 & $5.5 \times 10^{-6}$ & $1.6 \times 10^{-5}$ & - & - & - & - \\
\hline 9 & 0.109 & 0.123 & - & - & - & - \\
\hline
\end{tabular}




\section{node2vec parameters and tuning}

The node2vec algorithm has several parameters that can have great influence on the final node embeddings 5 and therefore the function prediction performance. For each species, we tuned these parameters for the experimental network and for the best combination of experimental and STRING edges separately. To do so, we employed a grid search. Below is a list of the parameters we tuned and the ranges of parameter values we considered:

- The number of random walks per node $([5,10,15,20,30])$,

- The parameter $p[5([0.1,0.5,1.0,1.5,3.0])$,

- The parameter $q[5]([0.1,0.5,1.0,1.5,3.0])$,

- The length of each random walk ([20,40,60,80,100,160]), and

- The dimensionality of the embedding vector $([32,64,96,128,150,200,500])$.

For each of the combinations above, we tried the $k \mathrm{NN}$ and ridge classifiers. The $k \mathrm{NN}$ also has one tunable parameter, the number of neighbors $k$, for which we considered the values $[1,2,5,7,9$, $11,15,21,31,51]$. The parameter $\lambda$ of ridge, which controls the amount of L2 regularization, was tuned among the values $[0.0001,0.001,0.01,0.1,0.5,1.0,2.0,3.0,5.0,10.0]$.

This amounts to 105,000 classification models per network per species. Each model was evaluated in each of the five cross-validation folds, by further subdividing the training set of each fold into a training ( $80 \%$ of original training set) and a validation set (20\% of originial training set). The combination of node2vec hyperparameters, classifier and classifier parameter that maximized the validation $\mathrm{F}_{\max }$ in each fold was subsequently trained on the entire training set (of the corresponding fold) and the resulting trained model was tested on the previously unseen test set. 


\section{Removing edges from the yeast EXP PPI network}

We started from the full EXP PPI network of yeast and step-wise removed $10 \%, 20 \%, 30 \%, \ldots$, $90 \%$ and $99 \%$ of its edges. The edges were removed at random, using two different strategies: In the first ("uniform"), the probability of removing an edge was uniform over all present edges. In the second ("degree"), it was inversely proportional to the smaller degree of the two nodes that the edge connects, so that nodes with the fewest edges are most likely to lose them. This procedure gave us 11 downsampled networks per sampling strategy on which we ran the GBA classifier, using the same cross-validation loop as before. We repeated this 5 times with different random seeds to obtain variability estimates. The performance is plotted against the amount of missing edges in Figure S2.

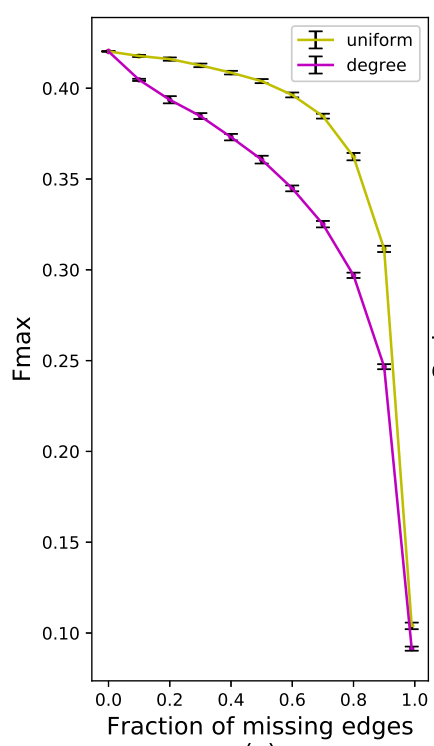

(a)

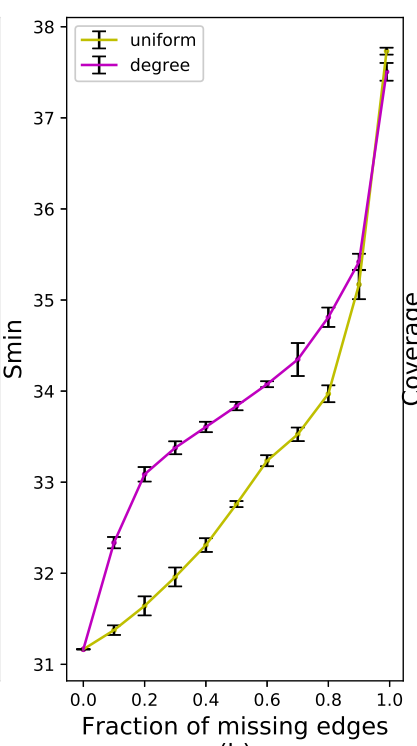

(b)

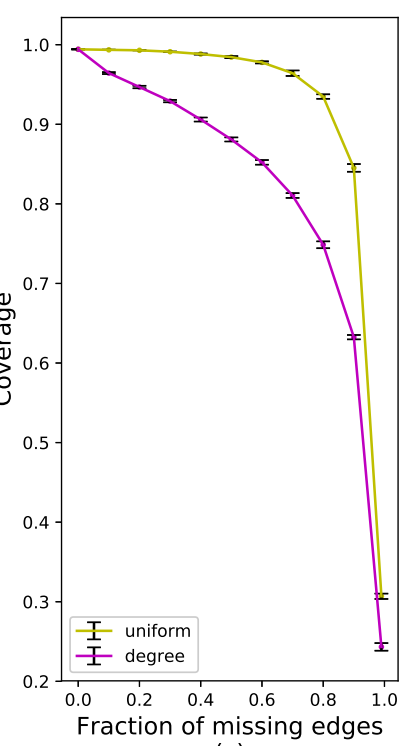

(c)

Figure $S 2: F_{\max }(a), S_{\min }(b)$ and coverage (c) of the GBA method on the yeast experimental PPI network ( $y$-axis) as a function of the fraction of missing edges ( $x$-axis). Edges where removed at random either uniformly (yellow) or inversely proportional to node degree, so that least connected nodes are more likely to lose their edges (purple). Error bars show standard deviation of 5 rounds of random sampling. 


\section{Using all STRING edges in a weighted graph}
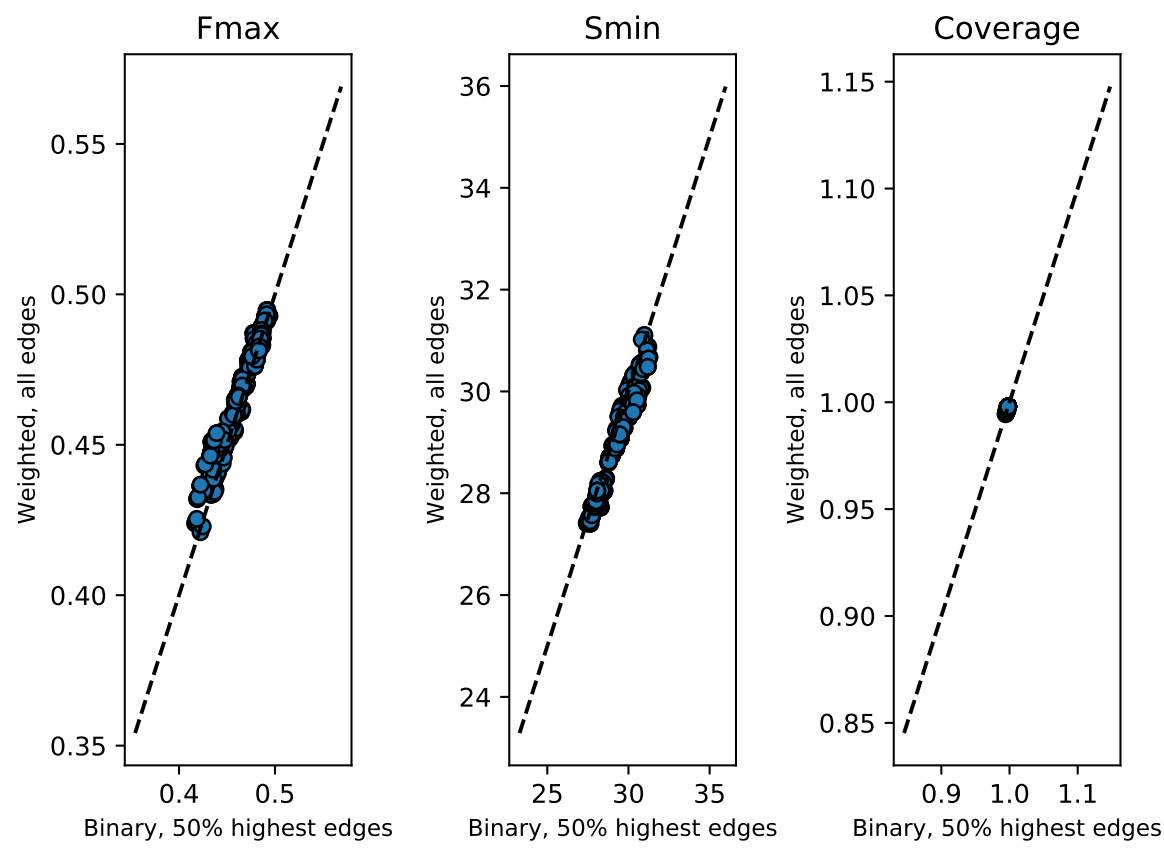

Figure S3: $\mathrm{F}_{\max }$ (left), $\mathrm{S}_{\min }$ (middle) and coverage (right) of the binary STRING networks with the $50 \%$ top edges ( $x$-axis) against the weighted ones with all edges ( $y$-axis) for $S$. cerevisiae. Each dot corresponds to one of the 511 combinations of the 9 STRING data sources added to the binary experimental network. The black dashed line shows the $y=x$ line to ease comparison.
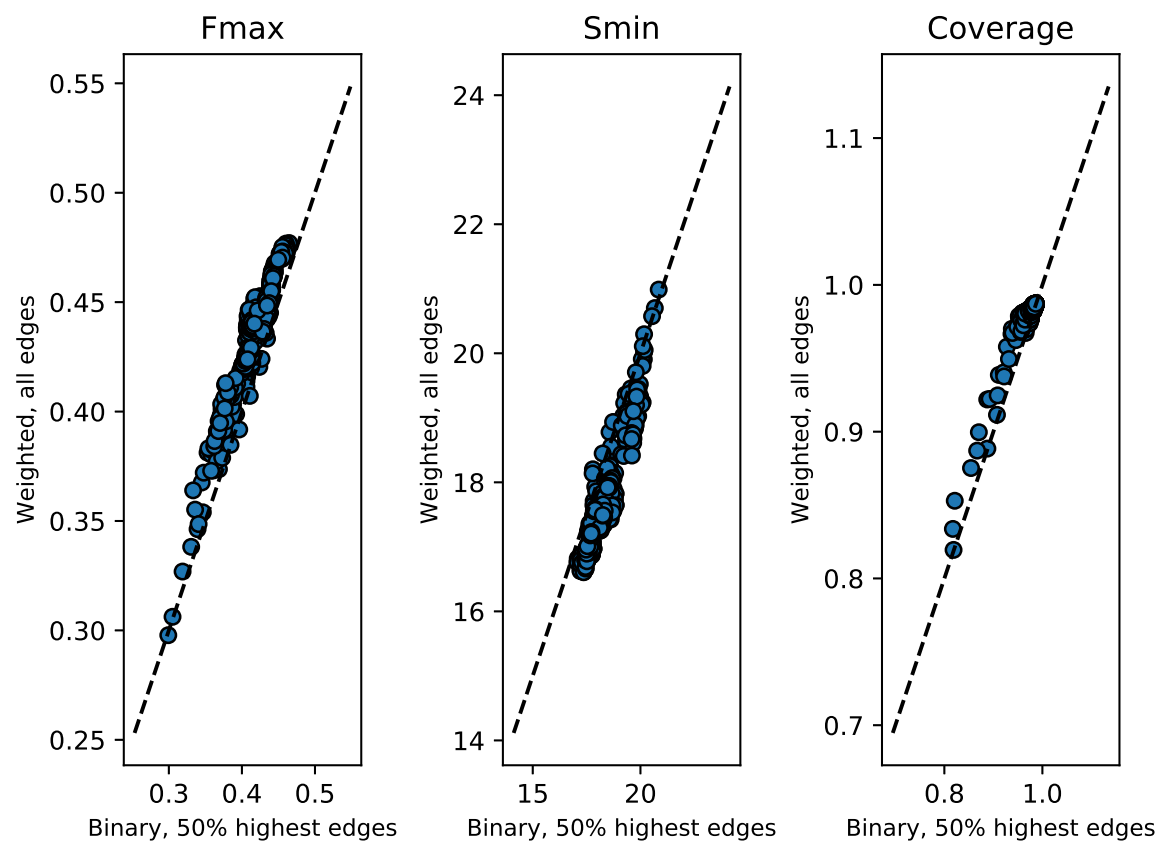

Figure S4: $\mathrm{F}_{\max }$ (left), $\mathrm{S}_{\min }$ (middle) and coverage (right) of the binary STRING networks with the $50 \%$ top edges ( $x$-axis) against the weighted ones with all edges ( $y$-axis) for E. coli. Each dot corresponds to one of the 511 combinations of the 9 STRING data sources added to the binary experimental network. The black dashed line shows the $y=x$ line to ease comparison. 

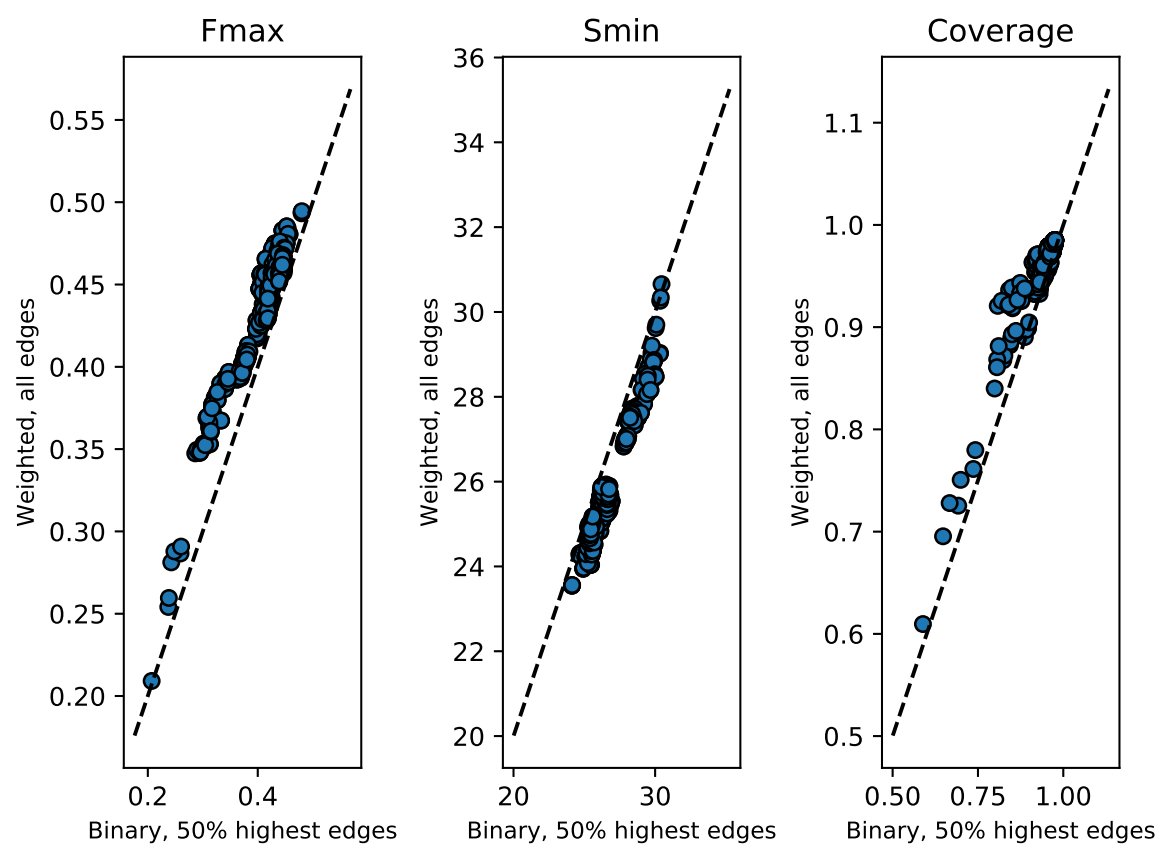

Figure S5: $\mathrm{F}_{\max }$ (left), $\mathrm{S}_{\min }$ (middle) and coverage (right) of the binary STRING networks with the $50 \%$ top edges ( $x$-axis) against the weighted ones with all edges ( $y$-axis) for A. thaliana. Each dot corresponds to one of the 511 combinations of the 9 STRING data sources added to the binary experimental network. The black dashed line shows the $y=x$ line to ease comparison.
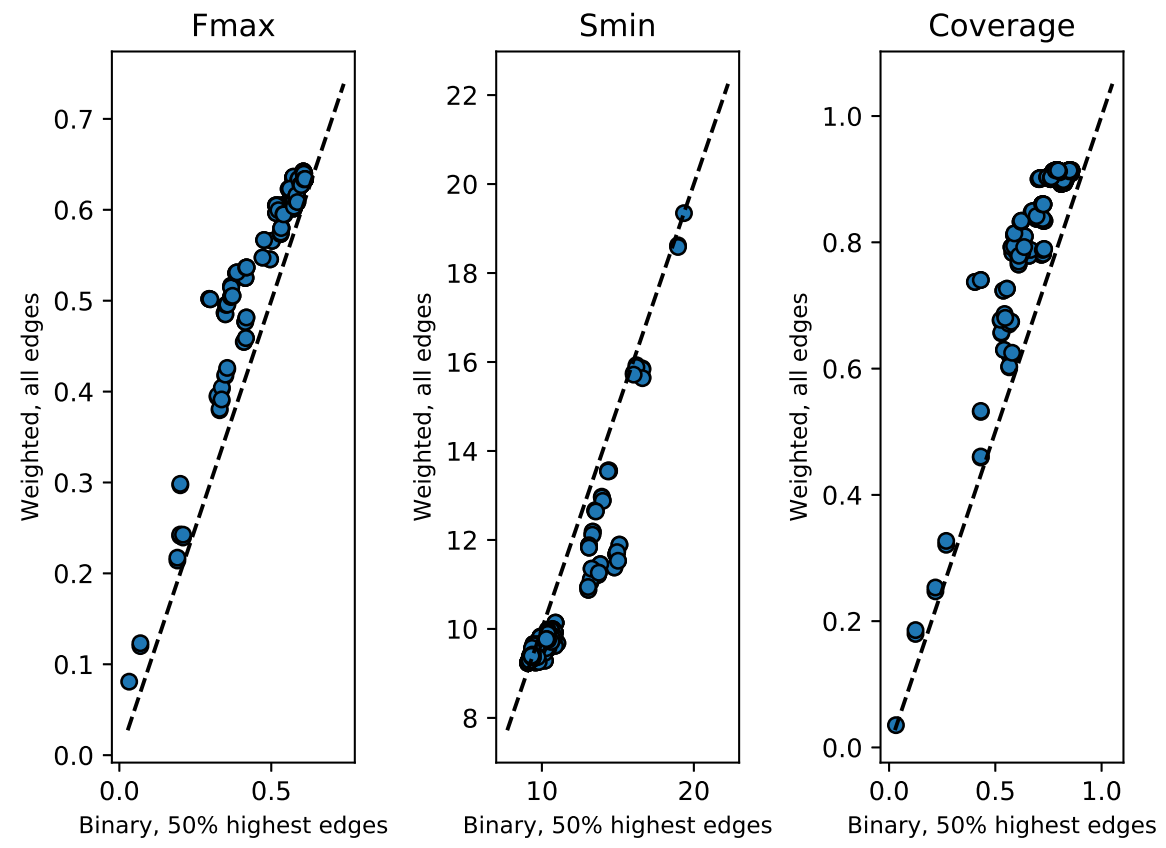

Figure S6: $\mathrm{F}_{\max }$ (left), $\mathrm{S}_{\min }$ (middle) and coverage (right) of the binary STRING networks with the $50 \%$ top edges ( $x$-axis) against the weighted ones with all edges (y-axis) for S. lycopersicum. Each dot corresponds to one of the 255 combinations of the 8 STRING data sources added to the binary experimental network. The black dashed line shows the $y=x$ line to ease comparison. 
Table S6: $\mathrm{F}_{\max }, \mathrm{S}_{\min }$ and coverage of the GBA method on the EXP+STRING PPI network in four species when using a binary network with the top $50 \%$ STRING edges or a weighted one with all STRING edges. The performance of the best combination for each metric and each species is shown. Statistically significant differences (paired t-test, FDR $<0.05$ ) are shown in bold.

\begin{tabular}{|c|l|l|l|l|l|l|}
\hline \multirow{2}{*}{ Species } & \multicolumn{2}{|c|}{$\mathrm{F}_{\max }$} & \multicolumn{2}{c|}{$\mathrm{S}_{\min }$} & \multicolumn{3}{c|}{ Coverage } \\
\hline & Binary & Weighted & Binary & Weighted & Binary & Weighted \\
\hline S. cerevisiae & $0.49 \pm 0.005$ & $0.49 \pm 0.005$ & $27.43 \pm 0.57$ & $27.40 \pm 0.61$ & $0.99 \pm 0.001$ & $0.99 \pm 0.001$ \\
\hline E. coli & $0.46 \pm 0.008$ & $\mathbf{0 . 4 8} \pm \mathbf{0 . 0 0 9}$ & $17.07 \pm 0.22$ & $16.61 \pm 0.35$ & $0.99 \pm 0.004$ & $0.99 \pm 0.003$ \\
\hline A. thaliana & $0.48 \pm 0.004$ & $\mathbf{0 . 4 9} \pm \mathbf{0 . 0 0 5}$ & $24.12 \pm 0.50$ & $\mathbf{2 3 . 5 4} \pm \mathbf{0 . 5 0}$ & $0.98 \pm 0.003$ & $\mathbf{0 . 9 9} \pm \mathbf{0 . 0 0 1}$ \\
\hline S. lycopersicum & $0.61 \pm 0.045$ & $0.64 \pm 0.043$ & $9.08 \pm 0.89$ & $9.23 \pm 1.00$ & $0.86 \pm 0.020$ & $\mathbf{0 . 9 1} \pm \mathbf{0 . 0 1 8}$ \\
\hline
\end{tabular}




\section{STRING performance per data source}

For every species, we tested all possible combinations of STRING data sources and found that certain combinations perform significantly better than others, as certain individual data sources were more informative (Fig S7, S9, ). To quantify the contribution of each data source we devised a statistical test described below.
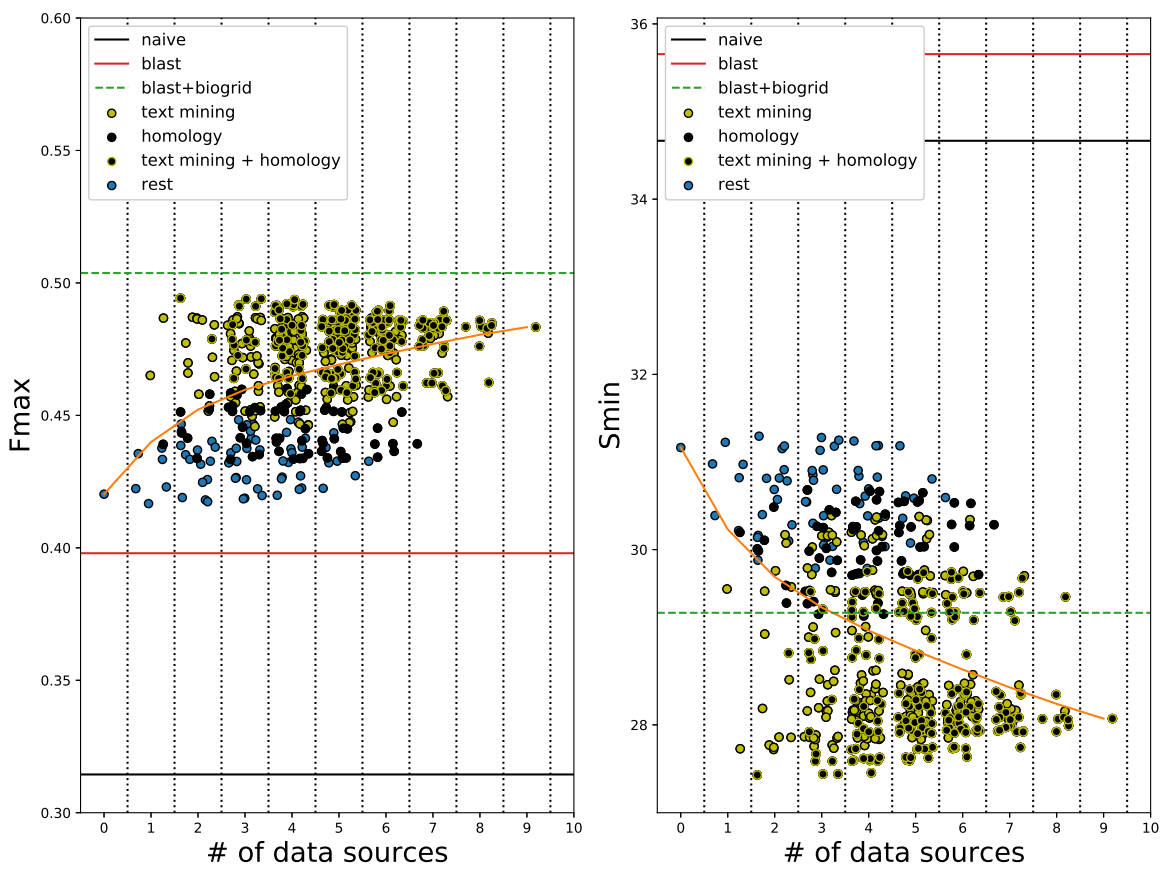

Figure S7: $\mathrm{F}_{\max }$ (left) and $\mathrm{S}_{\min }$ (right) in $S$. cerevisiae (y-axis) as a function of the number of STRING data sources included ( $x$-axis). Each dot corresponds to one combination of data sources added to the experimental network. Combinations that include "text mining" and/or "text mining transferred" are shown in yellow, combinations that include "homology" in black and combinations that include both in black with yellow border. The rest of the combinations are shown in blue. To ease visibility, we added a random number in the range $[-0.5,0.5]$ to each combination of the same number of sources. Zero data sources corresponds to the EXP network and the orange line shows the average performance for a specific number of data sources. Horizontal lines denote the performance of the naive (black), BLAST (red) and the combination of BLAST with the EXP PPI network (dashed green). 

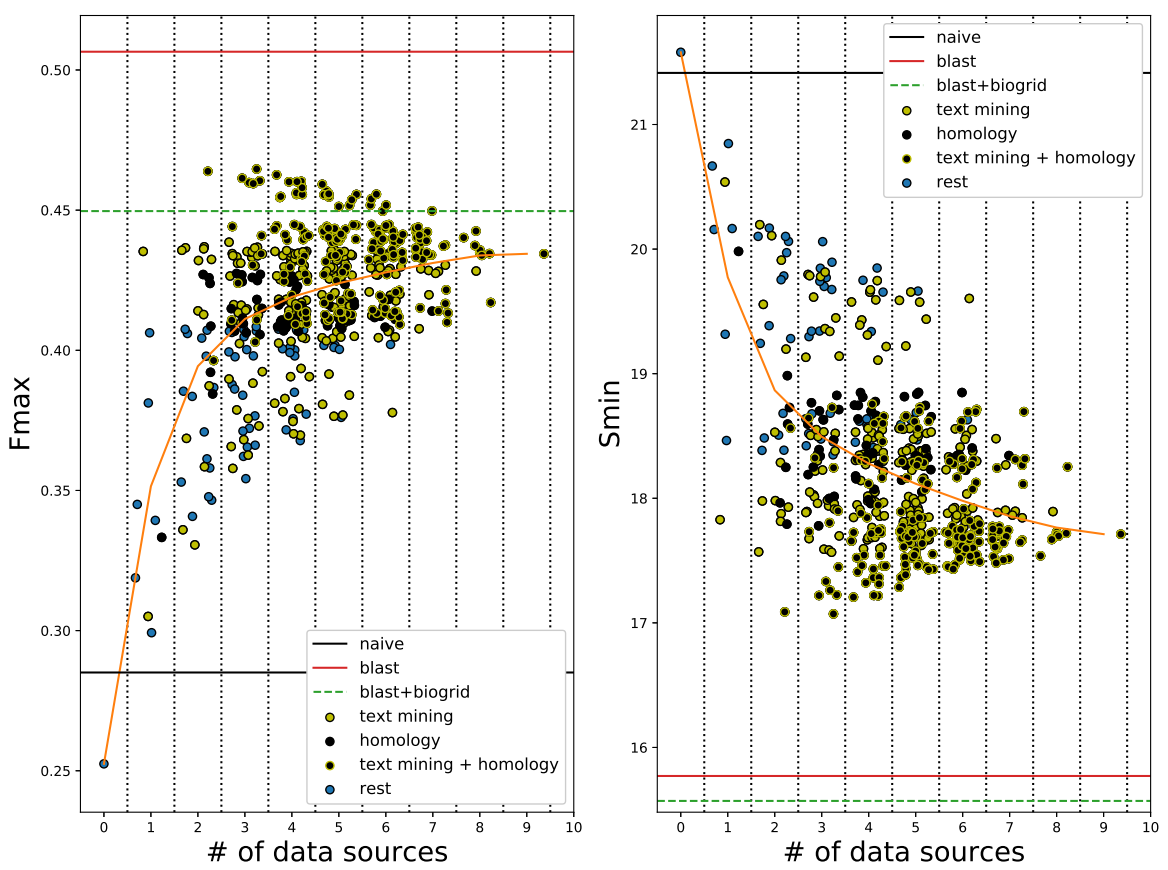

Figure $\mathrm{S} 8: \mathrm{F}_{\max }$ (left) and $\mathrm{S}_{\min }$ (right) in E. coli (y-axis) as a function of the number of STRING data sources included ( $x$-axis). Each dot corresponds to one combination of data sources added to the experimental network. Combinations that include "text mining" and/or "text mining transferred" are shown in yellow, combinations that include "homology" in black and combinations that include both in black with yellow border. The rest of the combinations are shown in blue. To ease visibility, we added a random number in the range $[-0.5,0.5]$ to each combination of the same number of sources. Zero data sources corresponds to the EXP network and the orange line shows the average performance for a specific number of data sources. Horizontal lines denote the performance of the naive (black), BLAST (red) and the combination of BLAST with the EXP PPI network (dashed green). 

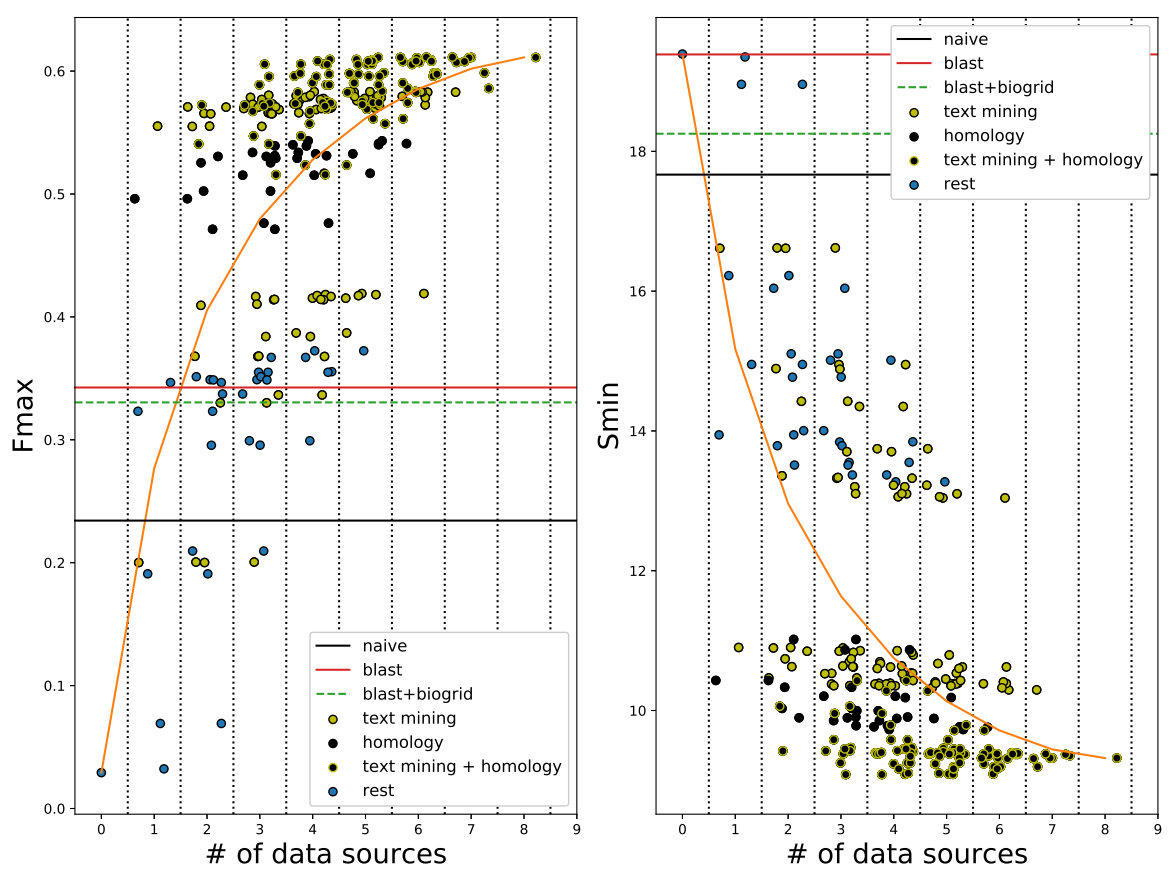

Figure S9: $\mathrm{F}_{\max }$ (left) and $\mathrm{S}_{\min }$ (right) in S. lycopersicum (y-axis) as a function of the number of STRING data sources included ( $x$-axis). Each dot corresponds to one combination of data sources added to the experimental network. Combinations that include "text mining" and/or "text mining transferred" are shown in yellow, combinations that include "homology" in black and combinations that include both in black with yellow border. The rest of the combinations are shown in blue. To ease visibility, we added a random number in the range $[-0.5,0.5]$ to each combination of the same number of sources. Zero data sources corresponds to the EXP network and the orange line shows the average performance for a specific number of data sources. Horizontal lines denote the performance of the naive (black), BLAST (red) and the combination of BLAST with the EXP PPI network (dashed green). 
For a species for which STRING contains protein associations from $N$ different data sources, there are $2^{N}-1$ ways to combine them (in combinations of different lengths). Each data source $i$ is present in $2^{N-1}$ of these combinations (denoted as $P_{i}$ ) and absent in the remaining ones (denoted as $\left.A_{i}\right)$. We define an "irreplaceability" score $(I R(i))$ for $i$ as the increase in the optimal performance of $P_{i}$ with respect to $A_{i}$. For $\mathrm{F}_{\max }$, this is formally defined in equation 12

$$
I R_{F}(i)=\max _{c \in P_{i}} F_{\max }(c)-\max _{c \in A_{i}} F_{\max }(c)
$$

An $I R_{F}(i)$ equal to 0 , means that the combination that achieves the maximum performance does not include data source $i$, which implies that it made redundant by other sources. A positive value for $I_{F}(i)$ means that $i$ is necessary for maximizing STRING performance and a negative value that $i$ is creating more errors than correct predictions. Since lower $S_{\min }$ means better performance, the definition of $I R$ for $\mathrm{S}_{\min }$ is slightly modified, so that a positive $I R$ still denotes that a data source is useful (equation 13 .

$$
I R_{S}(i)=\min _{c \in A_{i}} S_{\min }(c)-\min _{c \in P_{i}} S_{\min }(c)
$$

We compared the observed $I R_{F}$ and $I R_{S}$ values to what would be expected by chance to obtain a measure of statistical significance. To do so, we did a permutation test for which we randomly mixed the elements of $P_{i}$ and $A_{i}$ and calculated the $I R_{F}$ and $I R_{S}$ values again. We repeated this 10,000 times for each data source to obtain a null distribution of the two statistics and counted the fraction of times the permuted statistic was larger than the observed one. Note that this is a one-sided test, meaning that we only tested whether a data source was significantly irreplaceable, but not whether it was significantly leading to worse performance. We performed 90 tests in total, so we also applied multiple testing correction using the FDR method. The observed test statistics and the corresponding p-values are listed in Tables S7,S8 for yeast, S9,S10 for E. coli, S11, S12 for arabidopsis and $\mathrm{S} 13$ S14 for tomato.

Table S7: $I R_{F}$ scores of STRING data sources, corresponding raw p-values and p-values corrected for multiple tests for $S$. cerevisiae

\begin{tabular}{|c|c|c|c|}
\hline Data source & $I R_{F}$ & $\mathrm{p}$-value & corrected p-value \\
\hline coexpression & -0.0026 & 0.9989 & 1.0 \\
\hline neighborhood transferred & -0.0024 & 0.9933 & 1.0 \\
\hline coexpression transferred & -0.0081 & 1.0 & 1.0 \\
\hline experiments transferred & -0.0078 & 1.0 & 1.0 \\
\hline textmining & 0.0154 & $<10^{-4}$ & $<0.007$ \\
\hline cooccurence & -0.0004 & 0.8738900 & 1.0 \\
\hline fusion & -0.0002 & 0.75490 & 1.0 \\
\hline textmining transferred & -0.0022 & 0.9715 & 1.0 \\
\hline homology & 0.0007 & $<10^{-4}$ & $<0.007$ \\
\hline
\end{tabular}

Table S8: $I R_{S}$ scores of STRING data sources, corresponding raw p-values and p-values corrected for multiple tests for $S$. cerevisiae

\begin{tabular}{|c|c|c|c|}
\hline Data source & $I R_{S}$ & $\mathrm{p}$-value & corrected p-value \\
\hline coexpression & -0.1572 & 0.9832 & 1.0 \\
\hline neighborhood transferred & -0.1571 & 0.9707 & 1.0 \\
\hline coexpression transferred & -0.4923 & 1.0 & 1.0 \\
\hline experiments transferred & -0.4663 & 1.0 & 1.0 \\
\hline textmining & 1.3206 & $<10^{-4}$ & $<0.007$ \\
\hline cooccurence & -0.0105 & 0.7568 & 1.0 \\
\hline fusion & -0.0111 & 0.8798 & 1.0 \\
\hline textmining transferred & -0.1867 & 1.0 & 1.0 \\
\hline homology & 0.2914 & $<10^{-4}$ & $<0.007$ \\
\hline
\end{tabular}


Table S9: $I R_{F}$ scores of STRING data sources, corresponding raw p-values and p-values corrected for multiple tests for $E$. coli

\begin{tabular}{|c|c|c|c|}
\hline Data source & $I R_{F}$ & $\mathrm{p}$-value & corrected p-value \\
\hline neighborhood & -0.0042 & 0.9695 & 1.0 \\
\hline neighborhood transferred & -0.0198 & 1.0 & 1.0 \\
\hline coexpression transferred & -0.0046 & 0.9962 & 1.0 \\
\hline experiments transferred & -0.0021 & 0.8759 & 1.0 \\
\hline textmining & -0.0045 & 0.9919 & 1.0 \\
\hline cooccurence & -0.0232 & 1.0 & 1.0 \\
\hline fusion & 0.0008 & 0.4949 & 1.0 \\
\hline textmining transferred & 0.0303 & $<10^{-4}$ & $<0.007$ \\
\hline homology & 0.0261 & $<10^{-4}$ & $<0.007$ \\
\hline
\end{tabular}

Table S10: $I R_{S}$ scores of STRING data sources, corresponding raw p-values and p-values corrected for multiple tests for $E$. coli

\begin{tabular}{|c|c|c|c|}
\hline Data source & $I R_{S}$ & $\mathrm{p}$-value & corrected p-value \\
\hline neighborhood & -0.1456 & 0.9418 & 1.0 \\
\hline neighborhood transferred & -0.5245 & 1.0 & 1.0 \\
\hline coexpression transferred & -0.2496 & 0.9996 & 1.0 \\
\hline experiments transferred & -0.1348 & 0.8769 & 1.0 \\
\hline textmining & -0.1483 & 0.9839 & 1.0 \\
\hline cooccurence & -0.3763 & 1.0 & 1.0 \\
\hline fusion & 0.016 & 0.4938 & 1.0 \\
\hline textmining transferred & 0.6704 & $<10^{-4}$ & $<0.007$ \\
\hline homology & 0.4951 & $<10^{-4}$ & $<0.007$ \\
\hline
\end{tabular}

Table S11: $I R_{F}$ scores of STRING data sources, corresponding raw p-values and p-values corrected for multiple tests for $A$. thaliana

\begin{tabular}{|c|c|c|c|}
\hline Data source & $I R_{F}$ & $\mathrm{p}$-value & corrected p-value \\
\hline coexpression & -0.0297 & 1.0 & 1.0 \\
\hline neighborhood transferred & -0.0225 & 0.96600 & 1.0 \\
\hline coexpression transferred & -0.0324 & 1.0 & 1.0 \\
\hline experiments transferred & -0.0275 & 1.0 & 1.0 \\
\hline textmining & 0.0258 & 0.00010 & 0.0052 \\
\hline cooccurence & 0.0001 & 0.50100 & 1.0 \\
\hline fusion & 0.0002 & 0.25070 & 1.0 \\
\hline textmining transferred & -0.0252 & 0.9982 & 1.0 \\
\hline homology & 0.0534 & $<10^{-4}$ & $<0.007$ \\
\hline
\end{tabular}

Table S12: $I R_{S}$ scores of STRING data sources, corresponding raw p-values and p-values corrected for multiple tests for $A$. thaliana

\begin{tabular}{|c|c|c|c|}
\hline Data source & $I R_{S}$ & $\mathrm{p}$-value & corrected p-value \\
\hline coexpression & -0.9484 & 1.0 & 1.0 \\
\hline neighborhood transferred & -0.7883 & 0.9984 & 1.0 \\
\hline coexpression transferred & -1.2361 & 1.0 & 1.0 \\
\hline experiments transferred & -1.1204 & 1.0 & 1.0 \\
\hline textmining & 0.5247 & 0.0659 & 1.0 \\
\hline cooccurence & -0.0069 & 0.8801 & 1.0 \\
\hline fusion & -0.0066 & 0.7477 & 1.0 \\
\hline textmining transferred & -1.0243 & 1.0 & 1.0 \\
\hline homology & 1.9112 & $<10^{-4}$ & $<0.007$ \\
\hline
\end{tabular}


Table S13: $I R_{F}$ scores of STRING data sources, corresponding raw p-values and p-values corrected for multiple tests for tomato

\begin{tabular}{|c|c|c|c|}
\hline Data source & $I R_{F}$ & $\mathrm{p}$-value & corrected p-value \\
\hline neighborhood transferred & -0.0002 & 0.98540 & 1.0 \\
\hline coexpression transferred & 0.0019 & 0.00380 & 0.1938 \\
\hline experiments transferred & 0.0031 & $<10^{-4}$ & $<0.007$ \\
\hline textmining & 0.0128 & $<10^{-4}$ & $<0.007$ \\
\hline cooccurence & 0.0002 & 0.24900 & 1.0 \\
\hline fusion & 0.0000 & 0.75380 & 1.0 \\
\hline textmining transferred & 0.0254 & $<10^{-4}$ & $<0.007$ \\
\hline homology & 0.0281 & $<10^{-4}$ & $<0.007$ \\
\hline
\end{tabular}

Table S14: $I R_{S}$ scores of STRING data sources, corresponding raw p-values and p-values corrected for multiple tests for tomato

\begin{tabular}{|c|c|c|c|}
\hline Data source & $I R_{S}$ & $\mathrm{p}$-value & corrected p-value \\
\hline neighborhood transferred & -0.0029 & 0.93930 & 1.0 \\
\hline coexpression transferred & -0.0812 & 1.0 & 1.0 \\
\hline experiments transferred & -0.2280 & 1.0 & 1.0 \\
\hline textmining & 0.1536 & $<10^{-4}$ & $<0.007$ \\
\hline cooccurence & -0.0085 & 0.9875 & 1.0 \\
\hline fusion & 0.0000 & 0.75470 & 1.0 \\
\hline textmining transferred & 0.2691 & $<10^{-4}$ & $<0.007$ \\
\hline homology & 1.2114 & $<10^{-4}$ & $<0.007$ \\
\hline
\end{tabular}




\section{Removing the most informative data sources}

Table S15: Changes in $\mathrm{F}_{\max }$ of the combined EXP + STRING network, when removing text mining and/or homology edges

\begin{tabular}{|l|c|c|c|c|}
\hline Data sources & S. cerevisiae & E. coli & A. thaliana & S. lycopersicum \\
\hline All mining/text mining & $0.49 \pm 0.005$ & $0.46 \pm 0.008$ & $0.48 \pm 0.004$ & $0.61 \pm 0.045$ \\
\hline $\begin{array}{l}\text { No text } \\
\text { transferred }\end{array}$ & 0.066 & $0.43 \pm 0.013$ & $0.45 \pm 0.007$ & $0.54 \pm 0.053$ \\
\hline No homology & $0.49 \pm 0.004$ & $0.44 \pm 0.011$ & $0.43 \pm 0.006$ & $0.58 \pm 0.044$ \\
\hline $\begin{array}{l}\text { No text mining/text mining } \\
\text { transferred/homology }\end{array}$ & $0.45 \pm 0.006$ & $0.41 \pm 0.010$ & $0.35 \pm 0.004$ & $0.37 \pm 0.042$ \\
\hline
\end{tabular}

Table S16: Changes in $\mathrm{S}_{\min }$ of the combined $E X P+S T R I N G$ network, when removing text mining and/or homology edges

\begin{tabular}{|l|c|c|c|c|}
\hline Data sources & S. cerevisiae & E. coli & A. thaliana & S. lycopersicum \\
\hline All mining & $27.42 \pm 0.57$ & $17.07 \pm 0.22$ & $24.12 \pm 0.50$ & $9.08 \pm 0.89$ \\
\hline $\begin{array}{l}\text { No text mining/text mining } \\
\text { transferred }\end{array}$ & & $17.78 \pm 0.43$ & $24.65 \pm 0.59$ & $9.72 \pm 1.16$ \\
\hline No homology & $27.72 \pm 0.57$ & $17.57 \pm 0.26$ & $26.03 \pm 0.51$ & $10.30 \pm 0.84$ \\
\hline $\begin{array}{l}\text { No text mining/text mining } \\
\text { transferred/homology }\end{array}$ & $29.79 \pm 0.63$ & $18.35 \pm 0.22$ & $28.47 \pm 0.47$ & $13.27 \pm 0.65$ \\
\hline
\end{tabular}




\section{PIPR training and results}

Table S17: Effect of hyperparameters in training of PIPR for predicting yeast PPIs from the BIOGRID database.

\begin{tabular}{|l|c|c|c|}
\hline Optimizer & Learning Rate & Validation loss & Validation accuracy \\
\hline ADAM & 0.001 & 0.507 & 0.771 \\
\hline ADAM & 0.0001 & 0.540 & 0.754 \\
\hline SGD + learning rate scheduler & 0.01 & 0.525 & 0.751 \\
\hline SGD + learning rate scheduler & 0.001 & 0.547 & 0.737 \\
\hline
\end{tabular}

The originally trained PIPR model in yeast generalized poorly in Arabidopsis (accuracy of $51 \%$ on a balanced dataset). Therefore, we chose to train PIPR on Arabidopsis by using the original trained model as initial conditions. We trained using Stochastic Gradient Descent with learning rate 0.001 and early stopping based on the validation loss with patience of 40 epochs. As validation set, we randomly selected $10 \%$ of the data and as loss function the binary cross-entropy. We did not use RMSprop optimizer, which was used by the authors, as it produced unstable results.

Table S18: Comparison of function prediction performance in arabidopsis and tomato of edges predicted with PIPR trained in either yeast or arabidopsis

\begin{tabular}{|c|c|c|c|}
\hline Train species & Test species & $\mathrm{F}_{\max }$ & $\mathrm{S}_{\min }$ \\
\hline S. cerevisiae & A.thaliana & $0.29 \pm 0.004$ & $30.48 \pm 0.57$ \\
\hline A. thaliana & A.thaliana & $0.29 \pm 0.006$ & $30.16 \pm 0.29$ \\
\hline S. cerevisiae & S. lycopersicum & $0.33 \pm 0.016$ & $17.05 \pm 0.70$ \\
\hline A. thaliana & S.lycopersicum & $0.35 \pm 0.017$ & $16.24 \pm 0.66$ \\
\hline
\end{tabular}




\section{References}

[1] Christian von Mering et al. "STRING: Known and predicted protein-protein associations, integrated and transferred across organisms". In: Nucleic Acids Research (2005). ISSN: 03051048. DOI: $10.1093 / \mathrm{nar} / \mathrm{gki005}$

[2] Wyatt T. Clark and Predrag Radivojac. "Information-theoretic evaluation of predicted ontological annotations". In: Bioinformatics 29.13 (2013), pp. i53-i61. ISSN: 13674803. DOI: 10.1093/bioinformatics/btt228

[3] Naihui Zhou et al. "The CAFA challenge reports improved protein function prediction and new functional annotations for hundreds of genes through experimental screens". In: bioRxiv (2019). DOI: $10.1101 / 653105$.

[4] Predrag Radivojac et al. "A large-scale evaluation of computational protein function prediction". In: Nature Methods 10.3 (2013), pp. 221-227. ISSN: 1548-7091. DOI: 10.1038/nmeth. 2340, arXiv: 1510.05682

[5] Aditya Grover and Jure Leskovec. node2vec: Scalable Feature Learning for Networks. 2016. arXiv: 1607.00653 [cs.SI] 